Full Length Article

\title{
High LDL levels lessen bone destruction during antigen-induced arthritis by inhibiting osteoclast formation and function
}

\author{
G. Ascone ${ }^{a}$, I. Di Ceglie ${ }^{\mathrm{a}}$, B. Walgreen ${ }^{\mathrm{a}}$, A.W. Sloetjes ${ }^{\mathrm{a}}$, E. Lindhout ${ }^{\mathrm{b}}$, I. Bot ${ }^{\mathrm{c}}$, F.A.J. van de Loo $^{\mathrm{a}}$, \\ M.I. Koenders ${ }^{\mathrm{a}}$, P.M. van der Kraan ${ }^{\mathrm{a}}$, A.B. Blom ${ }^{\mathrm{a}}$, M.H.J. van den Bosch ${ }^{\mathrm{a}}$, P.L.E.M. van Lent ${ }^{\mathrm{a}, *}$ \\ ${ }^{a}$ Department of Experimental Rheumatology, Radboud University Medical Center, Radboud Institute of Molecular Life Sciences (RIMLS), Nijmegen, the Netherlands \\ ${ }^{\mathrm{b}}$ Future Diagnostics Solutions (FDx), Wijchen, the Netherlands \\ ${ }^{\mathrm{c}}$ Division of BioTherapeutics, Leiden Academic Centre for Drug Research (LACDR), Leiden, the Netherlands
}

\section{A R T I C L E I N F O}

\section{Keywords:}

High LDL levels

Bone destruction

oxLDL

Osteoclasts

ITAM co-stimulation pathway

\begin{abstract}
A B S T R A C T
Rheumatoid arthritis (RA) is a chronic inflammatory disease, characterized by severe joint inflammation and bone destruction as the result of increased numbers and activity of osteoclasts. RA is often associated with metabolic syndrome, whereby elevated levels of LDL are oxidized into oxLDL, which might affect osteoclastogenesis. In this study, we induced antigen-induced arthritis (AIA) in Apoe $e^{-/-}$mice, which spontaneously develop high LDL levels, to investigate the effects of high LDL/oxLDL levels on osteoclast differentiation and bone destruction. Whereas basal levels of bone resorption were comparable between naive WT and Apoe $e^{-1-}$ mice, induction of AIA resulted in a significant reduction of bone destruction in Apoe ${ }^{-/-}$mice as compared to WT controls. In line with that, the $\mathrm{TRAP}^{+}$area on the cortical bone was significantly decreased. The absence of Apoe did affect neither the numbers of CD11 $\mathrm{b}^{+} \mathrm{Ly} 6 \mathrm{C}^{\text {high }}$ and $\mathrm{CD} 11 \mathrm{~b}^{-} / \mathrm{Ly}_{6 \mathrm{C}^{\text {high }}}$ osteoclast precursors (OCPs) in the BM of naïve mice nor their in vitro osteoclastogenic potential as indicated by comparable mRNA expression of osteoclast markers. Addition of oxLDL, but not LDL, to pre-osteoclasts from day 3 and mature osteoclasts from day 6 of osteoclastogenesis strongly reduced the number of $\mathrm{TRAP}^{+}$osteoclasts and their resorptive capacity. This coincided with a decreased expression of various osteoclast markers. Interestingly, oxLDL significantly lowered the expression of osteoclast-associated receptor (Oscar) and the DNAX adaptor protein-12 encoding gene Tyrobp, which regulate the immunoreceptor tyrosine-based activation motif (ITAM) co-stimulation pathway that is strongly involved in osteoclastogenesis. Collectively, our findings suggest that under inflammatory conditions in the joint, high LDL levels lessen bone destruction during AIA, probably by formation of oxLDL that inhibits osteoclast formation and activity through modulation of the ITAM-signaling.
\end{abstract}

\section{Introduction}

Rheumatoid arthritis (RA) is a chronic autoimmune disease that affects the joints, and is characterized by synovial inflammation and excessive bone destruction [1,2]. Elevated production of pro-inflammatory mediators leads to increased levels of macrophage colonystimulating factor (M-CSF) and receptor activator of nuclear factor- $\mathrm{\kappa B}$ (RANK) ligand (RANKL), which induce the differentiation of myeloid precursors into osteoclasts $[3,4]$ that is accompanied by increased expression of osteoclast-specific markers [5-7]. In mice, particularly $\mathrm{CD}_{11} \mathrm{~b}^{+} \mathrm{Ly}_{6 \mathrm{C}^{\text {high }}}$ and $\mathrm{CD} 11 \mathrm{~b}^{-} \mathrm{Ly}^{\mathrm{L}} \mathrm{C}^{\text {high }}$ precursors in the bone marrow
(BM) are known to increase during experimental inflammatory arthritis and they are recruited to the inflamed joint where they can differentiate into osteoclasts $[8,9]$. Next to RANK/RANKL signaling, the balance between the immunoreceptor tyrosine-based activation motif (ITAM) and the immunoreceptor tyrosine-based inhibitory motif (ITIM) co-stimulatory signaling plays an important role in modulating osteoclastogenesis [10-12]. Within this system, osteoclast-associated receptor (OSCAR) and paired immunoglobulin-like receptor A (PIR-A) activate ITAM-signaling via association with Fc receptor common $\gamma$-chain $(\mathrm{FcR} \gamma)$, whereas the triggering receptor expressed on myeloid cells-2 (TREM-2) activates ITAM-signaling upon binding to DNAX adaptor

\footnotetext{
*Corresponding author at: Experimental Rheumatology, Route 272, Radboud University Medical Center, Geert Grooteplein 26-28, 6525GA Nijmegen, the Netherlands.

E-mail addresses: Giuliana.Ascone@radboudumc.nl (G. Ascone), Irene.DiCeglie@radboudumc.nl (I. Di Ceglie), Birgitte.Walgreen@radboudumc.nl (B. Walgreen), Annet.Sloetjes@radboudumc.nl (A.W. Sloetjes), Lindhout.E@future-diagnostics.nl (E. Lindhout), I.Bot@lacdr.leidenuniv.nl (I. Bot),

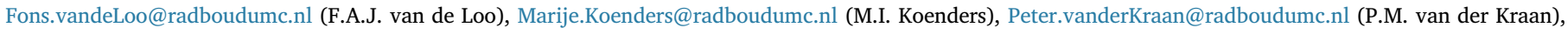
Arjen.Blom@radboudumc.nl (A.B. Blom), Martijn.vandenBosch@radboudumc.nl (M.H.J. van den Bosch), Peter.vanLent@radboudumc.nl (P.L.E.M. van Lent).
} 
protein of $12 \mathrm{kDa}$ (DAP12) [13,14]. Conversely, the immunoglobulinlike receptor B (PIR-B) signals through an ITIM motif and as such negatively regulates the ITAM-signaling, and thus osteoclastogenesis [15]. As the activation of the ITAM-pathway may further drive osteoclast differentiation and function, its regulation can be particularly relevant in inflammatory immune-based diseases like RA [10]. RA is often associated with metabolic syndrome, and both are characterized by elevated levels of low density lipoproteins (LDL) that are oxidized into oxLDL in inflammatory environments [16-18]. Therefore, the enhanced presence of oxLDL during inflammatory arthritis might affect differentiation and activation of osteoclasts, causing bone destruction in the arthritic joints. In order to investigate this, we used Apolipoprotein $\mathrm{E}$ deficient $\left(\right.$ Apoe $\left.^{-/-}\right)$mice that are commonly used as a model for hypercholesterolemia [19]. Apolipoprotein E (Apoe) is a lipoprotein that regulates LDL transportation and its absence strongly elevates circulating LDL levels in humans as well as in mice [20]. In this study, we elicited antigen-induced arthritis (AIA) in knee joints of $\mathrm{Apoe}^{-/-}$mice and their wild type (WT) controls to investigate the effects of high LDL levels and their oxidation to oxLDL, increased by the inflammatory milieu, on osteoclast-mediated bone destruction. Further, using in vitro experiments, we aimed at identification of molecular pathways involved in the effects of LDL/oxLDL on osteoclast differentiation and activation.

\section{Methods}

\subsection{Animals}

B6.129P2-Apoe $e^{\text {tm1Unc }} / J$ mice (JAX strain) and wild type C57Bl/6J controls were obtained from the Charles River Laboratory (Leiden, the Netherlands). All mice were housed in filter-top cages and received a standard chow diet and acidified water ad libitum. Male mice between 10 and 12 weeks were used in all the experiments, which were performed in accordance with the Dutch regulations and guidelines for care and use of laboratory animals. All animal studies were approved by the Radboud University's Animal Experiment Committee, Nijmegen-the Netherlands (RU-DEC 2014-191).

\subsection{Induction of antigen-induced arthritis}

Mice were immunized with $100 \mu \mathrm{g} / \mathrm{ml}$ of methylated bovine serum albumin (mBSA; Sigma-Aldrich) emulsified in $100 \mu$ l Freund's complete adjuvant (CFA). Injections were divided over the flanks and the footpad of the front paws. Heat killed Bordetella Pertussis was administered as an additional adjuvant intraperitoneally. One week later, two subcutaneous injections of $50 \mu \mathrm{g}$ of mBSA/CFA were given as a booster in the neck region. Two weeks after these booster injections, arthritis was induced in the right knee joint by intra-articular injection of $60 \mu \mathrm{g}$ of mBSA in $6 \mu l$ saline.

\subsection{Histology}

Total knee joints were dissected, fixed in phosphate-buffered formalin ( $\mathrm{pH} 7.4$ ), decalcified in ethylenediaminetetraacetic acid (EDTA) and subsequently embedded in paraffin wax. Coronal sections of $7 \mu \mathrm{m}$ of various depths, representing the whole knee joint, were stained with hematoxylin \& eosin (H\&E) for histological analysis. Bone destruction was evaluated in 13 well-defined areas of the knee joint (shown in Supplementary File 1) and scored arbitrarily on a scale from 0 (no erosion) to 3 (connection between joint cavity and bone marrow). Total knee joint sections were stained for tartrate-resistant acid phosphatase (TRAP), using the Leukocyte Acid Phosphatase Kit (Sigma-Aldrich) according to the manufacturer's protocol. The amount of $\mathrm{TRAP}^{+}$area present along the cortical bone and trabecular bone within the bone marrow (BM) cavity, representing osteoclasts, was measured in the arthritic joints as percentage (\%) of positive area above a fixed threshold using Leica Application Suite software (Leica Microsystems).

\subsection{Flow cytometry}

Numbers of osteoclast precursors in the BM were determined with flow cytometry. Bone marrow was isolated from femurs and tibias of mice by flushing the marrow cavity with medium and passing the cell suspension through a strainer. After lysis of erythrocytes, bone marrow cells were incubated with Fc-blocking antibody (BD Pharmingen antimouse CD16/CD32, clone 2.4G2; BD Biosciences, San Jose, CA, USA), followed by staining with the following mix of antibodies: CD11bfluorescein isothiocyanate (FITC), CD90.2-phycoerythrin (PE), CD45R/ B220-PE, CD49b-PE, NK1.1-PE, Ly6G-PE, Ly6C-allophycocyanin-cyanine 7 (APC-Cy7), and F4/80-PE-Cy7 (all BD Biosciences). Samples were acquired with a Gallios flow cytometer (Beckman Coulter Life Sciences, Indianapolis, IN, USA).

\subsection{In vitro osteoclast differentiation}

Following BM isolation, total BM cells were seeded into 96-well plates at a density of $10^{5}$ cells/well and cultured up to 7 days in $100 \mu \mathrm{l}$ of $\alpha$-minimum essential medium (Thermo Fisher Scientific), supplemented with $5 \%$ FCS, penicillin/streptomycin, $30 \mathrm{ng} / \mathrm{ml}$ recombinant mouse (rm)M-CSF, and $20 \mathrm{ng} / \mathrm{ml}$ rmRANKL (R\&D Systems). Culture medium was refreshed after 3 days.

\section{6. oxLDL preparation and stimulation of osteoclasts}

LDL was isolated by single-spin density gradient ultracentrifugation from EDTA-treated blood from healthy volunteers and oxidized as previously described [21]. At various time-points (day 3, 5 or day 6) of osteoclastogenesis, preosteoclasts or yet mature osteoclasts were stimulated with either $10 \mu \mathrm{g} / \mathrm{ml}$ oxLDL or $10 \mu \mathrm{g} / \mathrm{ml} \mathrm{LDL-C} \mathrm{as} \mathrm{control} \mathrm{and}$ the medium was refreshed every 3 days. This concentration was chosen to resemble the LDL-C levels in vivo [22].

\subsection{RNA isolation and $q R T-P C R$}

RNA from osteoclast cultures was isolated with TRIzol reagent (Sigma-Aldrich) at different time-points during osteoclastogenesis (days $0,3,4,6$ and 7). Briefly, mRNA concentration was measured with a Nanodrop spectrophotometer and subsequently reverse-transcribed into complementary DNA (cDNA) using oligo (dT) primers. qRT-PCR was performed using the Applied Biosystems StepOnePlus RT-PCR System (Thermo Fisher Scientific). Specific primer sequences used to assess gene expression are listed in Table 1. Glyceraldehyde 3-phosphate dehydrogenase (Gapdh) was used as housekeeping gene. Samples were normalized for the expression of Gapdh by calculating the comparative threshold: $-\Delta \mathrm{Ct}=-\left(\mathrm{C}_{\mathrm{t}}\right.$ gene of interest $-\mathrm{C}_{\mathrm{t}}$ Gapdh $)$.

\subsection{Determination of TRAP activity in the supernatants}

Culture supernatants were collected after 7 days of differentiation and TRAP activity was measured with a colorimetric assay. Briefly, $p$ nitrophenyl phosphate (NewEngland Biolabs) was diluted in buffer containing $420 \mathrm{mM}$ acetic acid (Sigma-Aldrich) and $160 \mathrm{mM}$ tartrate solution (Merck) and added 1:1 to culture supernatant. After $1 \mathrm{~h}$, the reaction was stopped with $0.5 \mathrm{M} \mathrm{NaOH}$ (Sigma-Aldrich) and the absorbance at $405 \mathrm{~nm}$ was determined using a spectrophotometric plate reader (Bio-Rad Laboratories).

\subsection{Resorption assay on calcium phosphate-coated plates}

Bone marrow cells were seeded at a density of $10^{5}$ cells/well onto 96-well biomimetic hydroxyapatite-like-coated plates that were prepared as previously described [23]. Preosteoclasts or mature osteoclasts 
Table 1

\begin{tabular}{|c|c|}
\hline Gene & PRIMER SEQUENCE (5'-3') \\
\hline \multirow[t]{2}{*}{ Gapdh } & FW: GGCAAATTCAACGGCACA \\
\hline & RV: GTTAGTGGGGTCTCGCTCCTG \\
\hline \multirow[t]{2}{*}{ Nfatc1 } & FW: ATGCGAGCCATCATCGA \\
\hline & RV: GGGATGTGAACTCGGAAGAC \\
\hline \multirow[t]{2}{*}{ Acp5 } & FW: GACAAGAGGTTCCAGGAG ACC \\
\hline & RV: GGGCTGGGGAAGTTCCAG \\
\hline \multirow[t]{2}{*}{ Calcr } & FW: CGTTCTTTATTACCTGGCTCTTGTG \\
\hline & RV: TCTGGCAGCTAAGGTTCTTGA AA \\
\hline \multirow[t]{2}{*}{ Мтр9 } & FW: GGAACTCACACGACATCTTCCA \\
\hline & RV: GAAACTCACACGCCAGAAGAATTT \\
\hline \multirow[t]{2}{*}{$\mathrm{Ca} 2$} & FW: GCTGCAGAGCTTCACTTGGT \\
\hline & RV: AAACAGCCAATCCATCCGGT \\
\hline \multirow[t]{2}{*}{ Oscar } & FW: TGGTCATCAGTTTCGAAGGTTCT \\
\hline & RV: CAGCCCCAAACGGATGAG \\
\hline \multirow[t]{2}{*}{ Dcstamp } & FW: TGTATCGGC TCATCTCCTCCAT \\
\hline & RV: GACTCCTTGGGTTCCTTGCTT \\
\hline \multirow[t]{2}{*}{ Clcn7 } & FW: AGCCTGGACTATGACAACAGC \\
\hline & RV: GGAAAGCCGTGTGGTTGATT \\
\hline \multirow[t]{2}{*}{ Ctsk } & FW: GAAGCAGTATAACAGCAAGGTGGAT \\
\hline & RV: TGTCTCCCAAGTGGTTCATGG \\
\hline \multirow[t]{2}{*}{ Fcgr1 } & FW: ACACAATGGTTTATCAACGGA \\
\hline & RV: TGGCCTCTGGGATGCTATAAC \\
\hline \multirow[t]{2}{*}{$F c g r 2 b$} & FW: GACAGCCGTGCTAAATCTTGCT \\
\hline & RV: GTGTCACCGTGTCTTCCTTGAG \\
\hline \multirow[t]{2}{*}{ Fcgr3 } & FW: GACAGGCAGAGTGCAGCTCTT \\
\hline & RV: TGTCTTCCTTGAGCACCTGGAT \\
\hline \multirow[t]{2}{*}{ Fcgr 4} & FW: TCTGGCATTCAAGCTGGTCTC \\
\hline & RV: TAGTTGGCATCCTGGTGTGG \\
\hline \multirow[t]{2}{*}{ Adgre1 } & FW: ACTGTGGAAAGCACCATGTTAG \\
\hline & RV: GCTGCCAAGTTAATGGACTCA \\
\hline \multirow[t]{2}{*}{ Tnfrsf11a } & FW: GCCCAGTCTCATCGTTCTGC \\
\hline & RV: GCAAGCATCATTGACCCAATTC \\
\hline \multirow[t]{2}{*}{ Tyrobp } & FW: TCTGGGTGACTTGGTGTTGAC \\
\hline & RV: CTGACCCTGAAGCTCCTGATAA \\
\hline \multirow[t]{2}{*}{ Trem2 } & FW: GCACCAACTTCAGATCCTCACT \\
\hline & RV: GCATGCAGGCTGGATTGACT \\
\hline \multirow[t]{2}{*}{ Il $2 \mathrm{rg}$} & FW: CTGTCTACACGGGCCTGAAC \\
\hline & RV: GCCAAGCACGTCTGTTCTGA \\
\hline \multirow[t]{2}{*}{ Pira } & FW: TACAGGAGGGTCGGGTGTAT \\
\hline & RV: GCATGCTTTCCAGGAGGTCT \\
\hline \multirow[t]{2}{*}{ Pirb } & FW: TTATGCCCAGGTGAAACCCT \\
\hline & RV: AGATTCGGCAGCCTGATTGTT \\
\hline \multirow[t]{2}{*}{ Csf1r } & FW: TGTCATCGAGCCTAGTGGC \\
\hline & RV: CGGGAGATTCAGGGTCCAAG \\
\hline
\end{tabular}

were stimulated with LDL/oxLDL starting from day 3 and day 5, respectively, and the medium was refreshed every 3 days. At day 10 the medium was removed from the wells, then $100 \mu \mathrm{l} /$ well of distilled water was added to lyse the cells. To analyze pit-formation the coated layer was stained with a Von Kossa staining. Briefly, a 5\% aqueous silver nitrate was added and incubated in the dark. Afterwards, wells were washed with water and incubated with $5 \%$ sodium carbonate in $4 \%$ formalin. Digital images were obtained with a camera mounted on an inverted-light microscope. The percentage of resorbed bone area was quantified using the Leica Application Suite software (Leica Microsystems).

\subsection{Statistics}

Statistics was performed using GraphPad Prism version 5.0 (GraphPad Software Inc.). Differences between two groups were tested using a two-tailed Student's $t$-test for comparing parametric variables, Mann-Whitney test for non-parametric variables (e.g. histological score) and multiple comparisons were tested using One-way ANOVA followed by Bonferroni's multiple comparison test. $P$ values $<0.05$ were considered significant. Data are presented as the mean \pm SEM.

\section{Results}

\subsection{Apoe $e^{-/-}$mice show reduced bone destruction during AIA}

First, we determined bone destruction in knee joints of hypercholesterolemic $A p o e^{-/-}$mice and their WT controls during AIA. Histological analysis showed less severe bone erosion in arthritic Apoe ${ }^{-1-}$ mice as compared to their arthritic WT controls (Fig. 1a). Quantification showed a significant reduction (by 25\%) in bone erosion in $A p o e^{-/-}$mice (Fig. 1b) when compared to WT mice. Only minor but comparable bone erosion was observed in naive joints of WT and Apoe $^{-/-}$mice (Fig. 1c and d).

\subsection{Arthritic Apoe ${ }^{-/-}$mice develop less osteoclasts along the cortical bone}

Because we found less bone destruction in $A p o e^{-/-}$mice during AIA, we next determined by enzymehistochemical TRAP staining whether this was due to a lower number of multinucleated resorbing osteoclasts. In line with the decreased bone destruction, at day 21 of AIA we observed a significant reduction in the amount of TRAP $^{+}$area (1.9-fold decrease) as well as the number of osteoclasts along the cortical bone of Apoe $e^{-/-}$mice when compared to WT controls (Fig. 2b and c). Further, we observed a different morphology of the osteoclasts present on the cortical bone, with the Apoe ${ }^{-/-}$cells being more flattened as compared to WT control cells (Fig. 2a).

3.3. Apoe deficiency does not affect the number of osteoclast precursors in the bone marrow and their osteoclastogenic potential

Next, we determined whether the absence of Apoe by itself could be the direct cause of the observed reduction in the number of osteoclasts. Flow cytometric analysis showed that the number of $\mathrm{CD} 11 \mathrm{~b}^{+} \mathrm{Ly} 6 \mathrm{C}^{\text {high }}$ and $\mathrm{CD} 11 \mathrm{~b}^{-}$Ly6 $\mathrm{C}^{\text {high }}$ cells, which are the main osteoclast precursors (OCPs) [8,9], were comparable in the BM of Apoe ${ }^{-/-}$and WT mice prior to induction of AIA. Interestingly, the percentage of CD11b ${ }^{-}$Ly6 $\mathrm{C}^{\text {high }}$ cells in the BM was even significantly increased in arthritic Apoe ${ }^{-/-}$mice (Fig. 3a and b). Next, we investigated whether the absence of Apoe itself altered their osteoclastogenic potential. We observed comparable kinetics in the expression of various osteoclast markers in differentiating osteoclasts from WT and Apoe ${ }^{-/-} \mathrm{BM}$ cells in vitro (Fig. 3c). Underlining this finding, no significant differences were observed in the number of TRAP ${ }^{+}$and multinucleated osteoclasts obtained from WT and Apoe ${ }^{-/-}$cultures $(58 \pm 10$ vs. $52 \pm 7$ osteoclasts, respectively) (Fig. 3d). Moreover, we found no difference in the amount of $\mathrm{TRAP}^{+}$area between arthritic WT and Apoe $e^{-/-}$mice in the trabecular bone within the BM cavity, which is not directly in contact with inflammatory mediators in the arthritic synovium (Fig. 3e and f). Together these data suggest that increased LDL oxidation in the inflammatory milieu rather than the Apoe deficiency in the arthritic joint may account for the differences in the number of osteoclasts on cortical bone.

3.4. oxLDL, but not $L D L$, blocks the differentiation of pre-osteoclasts into mature osteoclasts

Because the number of osteoclasts exclusively was reduced in the arthritic environment, we hypothesised that local inflammation may be involved. Apoe $e^{-/-}$mice spontaneously develop elevated levels of LDL, and this LDL is oxidized into oxLDL in inflamed areas. Therefore, we investigated whether LDL or oxLDL affected in vitro osteoclast differentiation. First, at day 3 of differentiation pre-osteoclasts were stimulated with oxLDL or LDL for $24 \mathrm{~h}$ and lipid uptake was detected by Oil Red $\mathrm{O}$ staining. As expected, oxLDL accumulated within the cells, whereas LDL did not (Fig. 4a). Pre-osteoclasts exposed to oxLDL for $24 \mathrm{~h}$ showed a significant decrease of the mRNA levels of various osteoclast markers. In contrast, the levels of Adgre1, the gene encoding for 

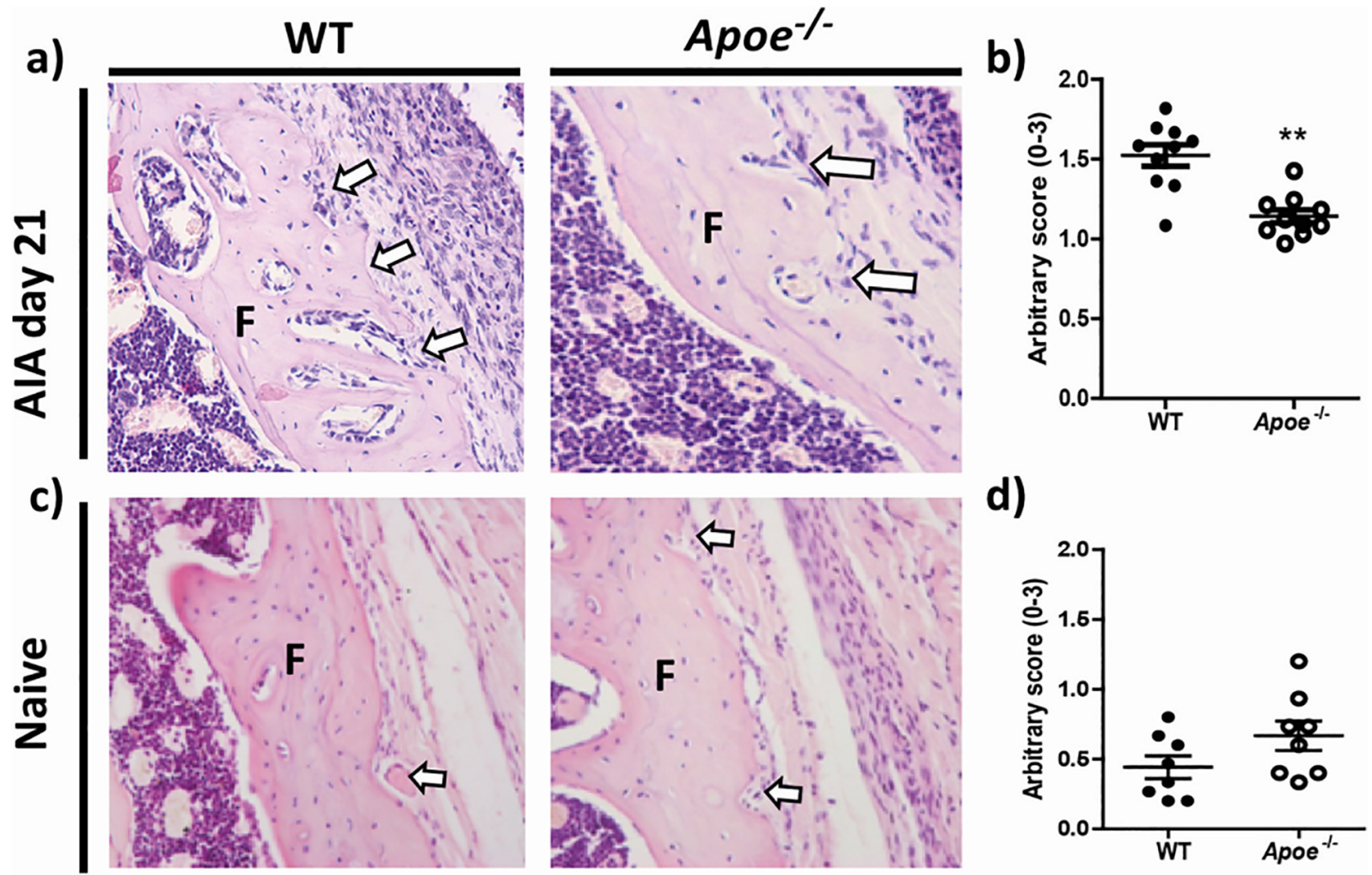

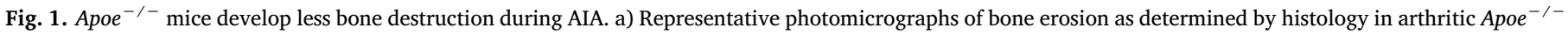

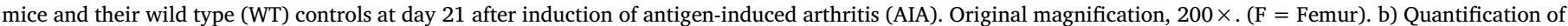

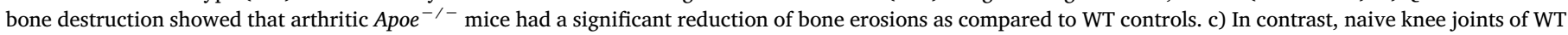

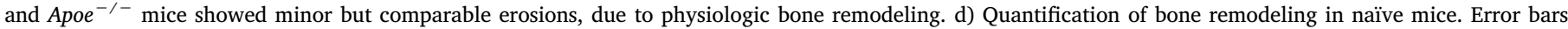
represent the mean \pm SEM of 10 mice (arthritic) and 8 mice (naïve). ( ${ }^{* *}=P<0.01$ versus WT controls).

F4/80 as macrophage marker remained significantly higher, underlining a blockage of osteoclast differentiation (Fig. 4b). Next, to assess the effects of oxLDL on osteoclast formation, the cells were further cultured up to 7 days. In agreement with our findings on the mRNA level, oxLDL, but not LDL, determined a nearly complete inhibition (94\% less than in control cells) of the number of TRAP ${ }^{+}$and multinucleated cells (Fig. 4c). This reduction was not due to cytotoxic effects of oxLDL, as indicated by the comparable fluorescence intensity signals after a DAPI staining among non-stimulated, LDL and oxLDL-stimulated cells (Fig. 4d). Although also apoptotic cells can internalize the DAPI stain qualitative assessment of the DAPI positive cells showed no significant differences in the nuclear morphology (data not shown), suggesting that oxLDL does not affect viability.

\section{5. oxLDL, but not $L D L$, inhibits the further fusion of multinucleated} osteoclasts and their capacity to break down the bone mineral matrix

At day 6, mature osteoclasts were stimulated for $24 \mathrm{~h}$ to investigate the effects of LDL and oxLDL at a later stage of differentiation. We found a significant reduction of $\mathrm{TRAP}^{+}$and multinucleated cells upon oxLDL exposure (46\% reduction as compared to control) (Fig. 5a and b). In accordance with the inhibition of osteoclastogenesis, we found that also osteoclast-mediated degradation of biomimetic hydroxyapatite-coated plates was strongly and significantly impaired by oxLDL both when pre-osteoclasts and mature osteoclasts were stimulated (Fig. 5c), indicating that exposure to oxLDL can inhibit the resorption machinery at different stages of osteoclastogenesis. Finally, as TRAP activity is commonly accepted as marker for bone resorption, we determined its secretion in the culture supernatants and found that exposure to oxLDL, but not LDL, of both pre-osteoclasts and mature osteoclasts significantly inhibited TRAP activity (Fig. 5d).
3.6. oxLDL strongly down-regulates the expression of molecules involved in the ITAM co-stimulation pathway

To identify a possible mechanism responsible for the effects of oxLDL on osteoclasts, we determined whether oxLDL affected the RANK/RANKL signaling, which is crucial to induce osteoclastogenesis. We found that oxLDL stimulation of pre-osteoclasts did not change the mRNA levels of Tnfrsf11 $a$ and Csf1r encoding genes for RANK and MCSFR, respectively (Fig. 6a). Next to RANK/RANKL signaling, factors involved in the co-stimulatory pathway play an important role in stimulating osteoclastogenesis and bone destruction. Therefore, we addressed whether oxLDL affected the expression of these factors. Interestingly, the mRNA of the immunoreceptor Oscar was strongly and significantly down-regulated in pre-osteoclasts by oxLDL. Although the expression of Trem2 was significantly increased, we found that the levels of Tyrobp, a gene that codes for DAP-12, which binds to TREM2, were significantly down-regulated upon oxLDL exposure. In contrast, the expression of other factors of the ITAM/ITIM co-stimulatory pathway such as Pira, Fcrg and Pirb - did not change upon oxLDL (Fig. 6b). Further, we assessed the expression of $\mathrm{Fc} \gamma$ receptors (Fc $\gamma \mathrm{Rs}$ ) as they are involved in ITAM-mediated induction of osteoclastogenesis and found that oxLDL significantly up-regulated the mRNA of Fcgr3, which has been associated with the inhibition of osteoclastogenesis [24] (Fig. 6c). These data suggest that at later stages of osteoclastogenesis, oxLDL does not affect either the RANK/RANKL signaling or the response to M-CSF-induced differentiation of pre-osteoclasts, but rather targets the ITAM co-stimulatory pathway to inhibit the further differentiation of pre-osteoclasts into mature and functional cells. 
a)
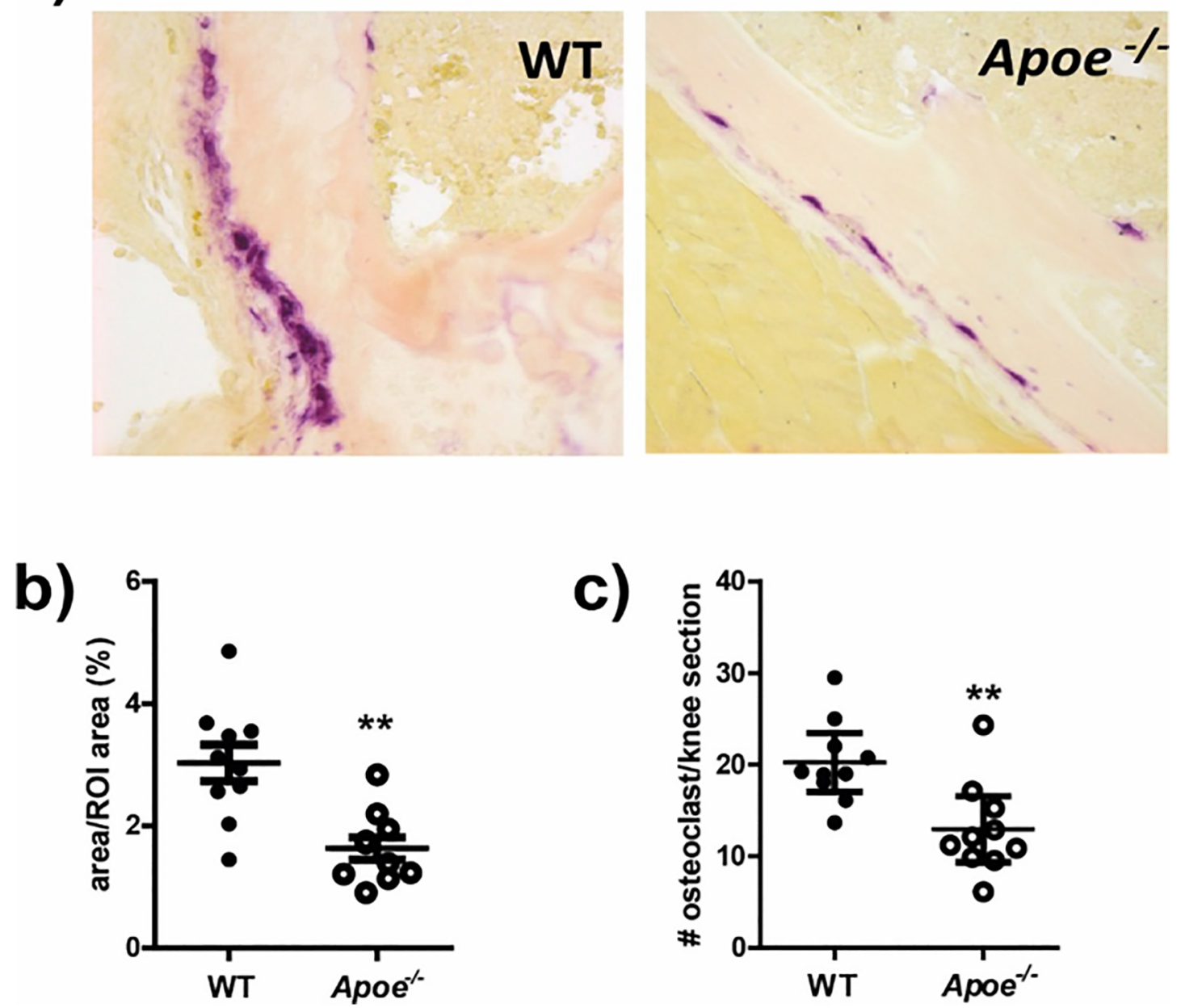

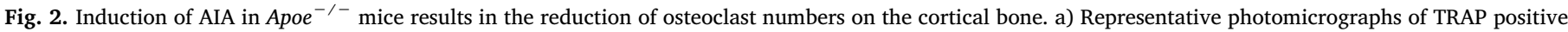

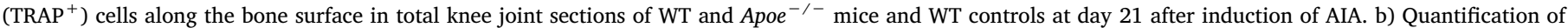

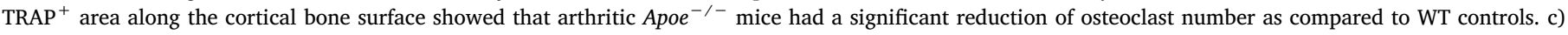

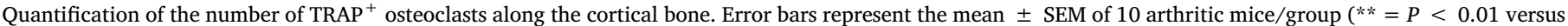
WT controls). Original magnification, $200 \times$.

\section{Discussion}

In the present study, we show that high LDL levels in $A p o e^{-/-}$mice in combination with local inflammation resulted in decreased bone destruction and reduction of the number of osteoclasts in the knee joints after local induction of AIA. Further, we show that oxLDL but not LDL prevents the differentiation of osteoclasts, and their function probably by decreasing the activity of the ITAM co-stimulatory pathway.

RA is often accompanied by dyslipidemia, characterized by high levels of LDL and low levels of high density lipoprotein (HDL) cholesterol $[25,26]$ and LDL has been proposed as a novel serum marker to predict radiographic progression in RA patients [27]. However the relation between high LDL levels and bone erosion in RA is still a matter of debate. In the serum transfer-induced arthritis (STIA) model Archer et al. found that $A p o e^{-/-}$mice fed a western-type diet (WD) developed enhanced synovial inflammation, although bone destruction was not affected [28]. In line with this, a previous study from our lab showed increased early ectopic bone formation in dyslipidemic mice during experimental osteoarthritis, likely due to the enhanced production of transforming growth factor-beta (TGF- $\beta$ ) by synovial macrophages $[29,30]$. TGF- $\beta$ strongly inhibits osteoclast formation and activity $[31,32]$. As we found that elevated levels of LDL and their increased oxidation during AIA in the Apoe ${ }^{-/-}$mice decreased bone destruction it can be that oxLDL acts as an anabolic factor by inducing the local production of TGF- $\beta$, thus decreasing osteoclast formation and bone destruction. Therefore, further studies including intra-articular injections of oxLDL will help to shed light on the effect of oxLDL on the development of joint destruction.

In RA patients the permeability of the endothelium is altered which allows a substantial access of LDL from the blood stream to the subendothelial layer [33] and joint tissues, and as such LDL levels in synovial fluids of RA patients resemble those in the blood [34]. After entering the inflamed joint, LDL becomes oxidized to oxLDL through the action of pro-inflammatory cytokines that stimulate the release of reactive oxygen species by inflammatory cells (ROS) [35-37]. Our findings show that hypercholesterolemic Apoe ${ }^{-/-}$mice developed less bone destruction owed to a substantial reduction of the number of osteoclasts in the arthritic knee joint. Apoe $e^{-/-}$mice are a widely accepted mouse model for hypercholesterolemia $[38,39]$. Apart from regulating LDL levels [40], APOE has been described as an inhibitor of various immune cells [41,42] and its absence may stimulate systemic inflammation thereby potentiating bone erosion. However, studying Apoe $^{-/-}$mice with high LDL levels, only physiological bone remodeling was found in naive knee joints not different from that in WT mice. In addition, in the absence of an inflammatory trigger, APOE did 

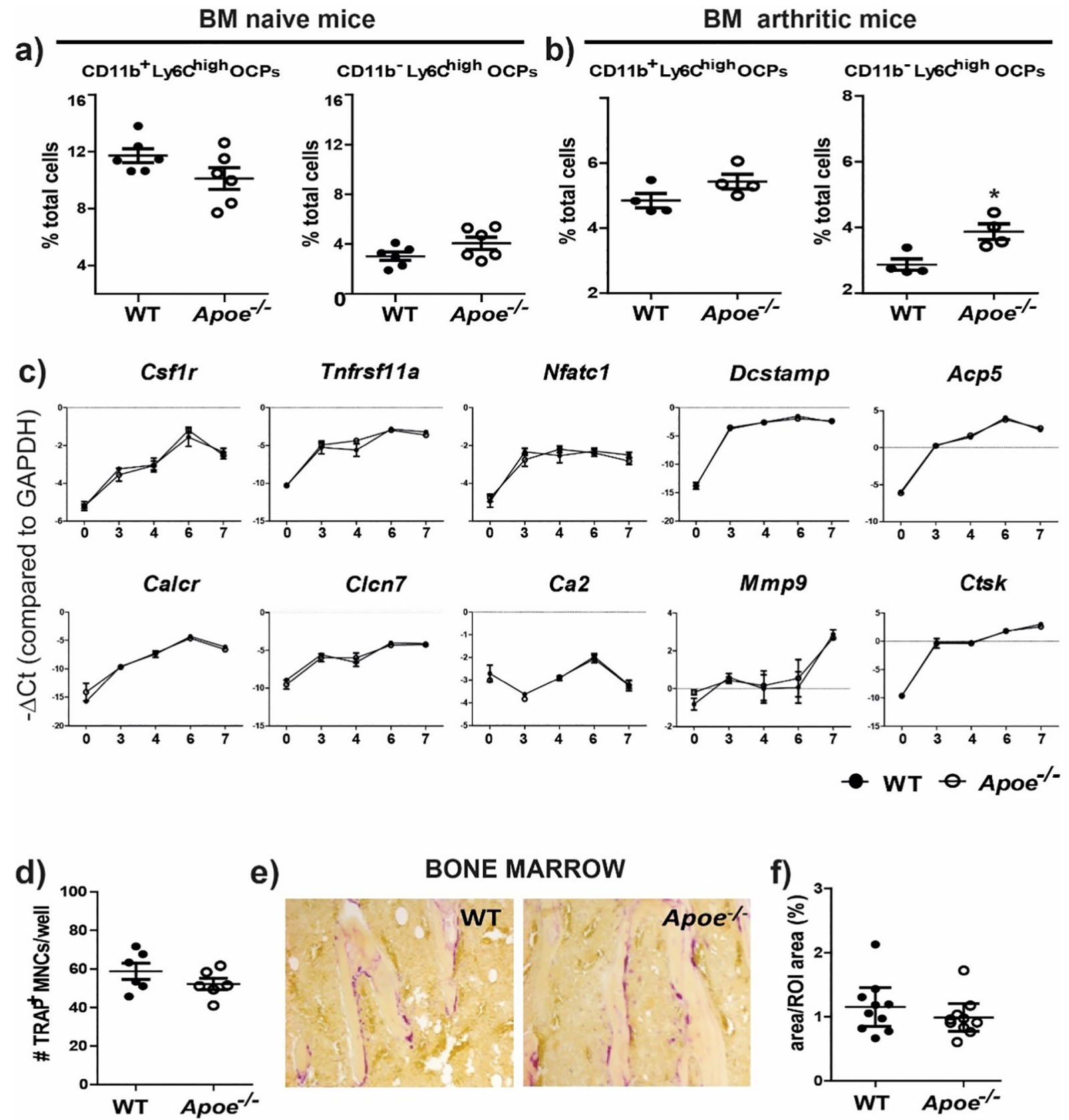

Fig. 3. The absence of Apoe does not affect the number of osteoclast precursors in the bone marrow or their osteoclastogenic potential. a) Comparable percentages of $\mathrm{CD} 11 \mathrm{~b}^{+} \mathrm{Ly}_{6 \mathrm{C}} \mathrm{high}^{\text {aig }}$ and $\mathrm{CD} 11 \mathrm{~b}^{-} \mathrm{Ly} 6 \mathrm{C}^{\text {high }}$ osteoclast precursors (OCPs) were present in the bone marrow (BM) of naïve wild type (WT) and Apoe $e^{-/-}$mice $(\mathrm{n}=6$ mice/ group). b) Notably, whereas we observed decreased numbers of osteoclasts at day 21 after AIA induction, Apoe ${ }^{-/-}$mice showed a significantly higher percentage (\%) of CD11 ${ }^{-}$Ly6 $\mathrm{C}^{\text {high }}$ OCPs compared to WT mice. c) Comparable messenger RNA (mRNA) expression levels of various osteoclast markers were determined at various time-points during osteoclastogenesis in WT and Apoe ${ }^{-/-}$bone marrow (BM) cells ( $\mathrm{n}=6$ mice/group). Colony stimulating factor 1 receptor (Csf1r); Tumor necrosis factor receptor superfamily member 11a (Tnfrsf11a); Nuclear factor of activated T cells 1 (Nfatc1); Dendritic cells specific transmembrane protein (Dcstamp); Acid phosphatase 5 (Acp5); Calcitonin receptor (Calcr); Chloride channel-voltage sensitive 7 (Clcn7); Carbonic anhydrase 2 (Ca2); Matrix metallopeptidase 9 (Mmp9); Cathepsin K (Ctsk); d) Quantification of TRAP ${ }^{+}$osteoclasts shows no differences in the number of osteoclasts. e) Moreover, as indicated in representative microphotographs, no differences in the amount of $\mathrm{TRAP}^{+}$osteoclasts were present in the trabecular bone within the BM. Original magnification, $200 \times$. f) Quantification of the $\mathrm{TRAP}^{+}$area within the BM expressed as percentage. Error bars represent the mean \pm SEM of 10 mice $(*=P<0.05$ compared to WT controls).

not affect the percentage of OCPs present in the bone marrow nor the kinetics of expression of osteoclast markers and their osteoclastogenic potential. This is in accordance with a previous study by Schilling et al. that described that mice lacking Apoe displayed an increased trabecular bone volume caused by an increased bone formation rate, probably due to undercarboxylation of osteocalcin that is an inhibitor of osteoblastogenesis [43]. Similarly, Nikolov et al. reported that Apoe $e^{-/-}$mice had higher cortical and trabecular bone mass than control WT mice at the basal level due to increased osteoblast -mediated bone formation [44]. However, it has to be noted that in contrast to our study in which we used young, 12-weeks old, male mice they used 20-weeks old female mice, suggesting that under hypercholesterolemic conditions both sex and age have an additional effect on bone metabolism. In our study we showed that neither high levels of LDL alone nor Apoe deficiency by itself, promote osteoclastogenesis and bone erosion. Further, increased LDL oxidation in arthritic Apoe $e^{-/-}$mice did not lead to a reduction of 
a)

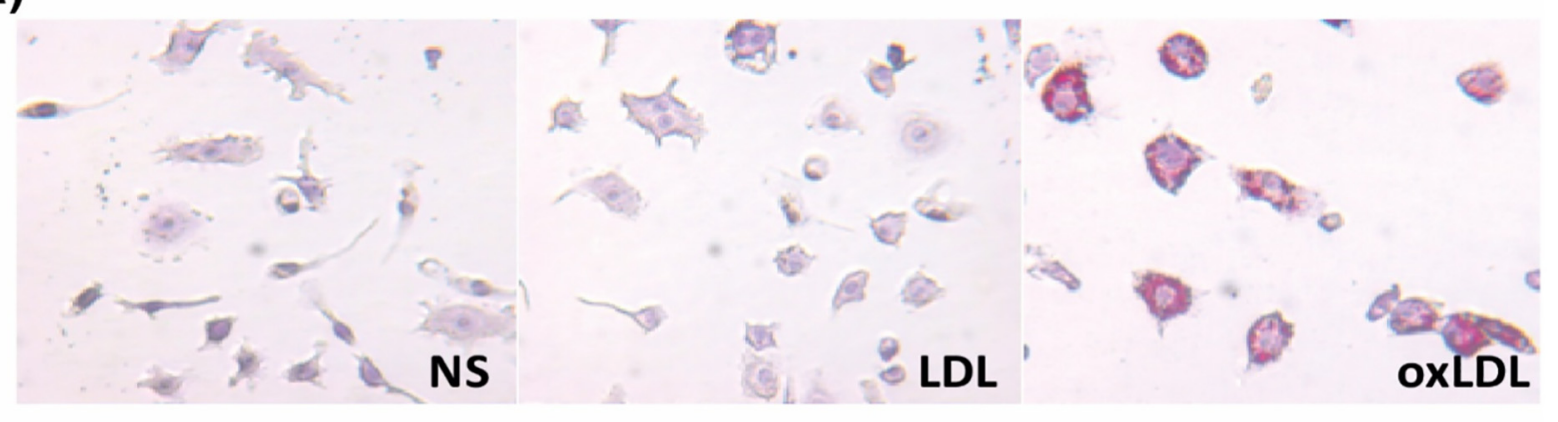

b) Dcstamp
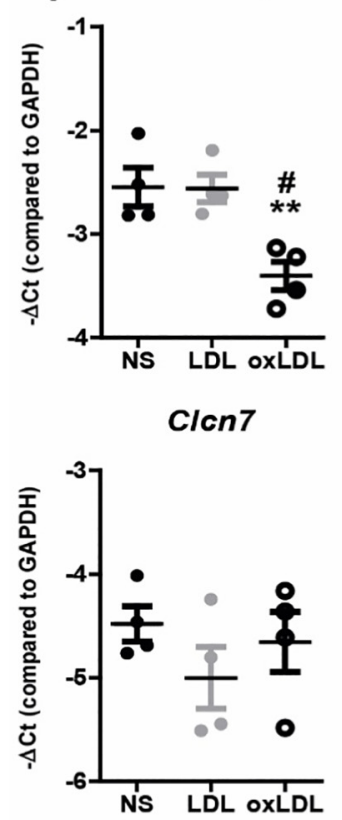

c)

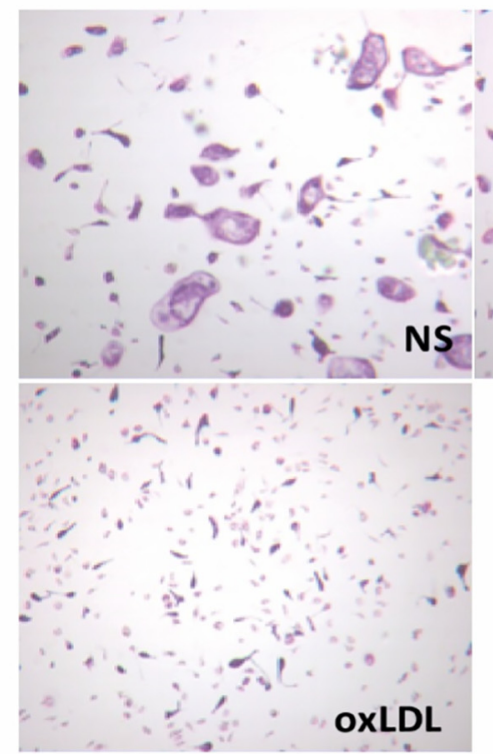

Acp5
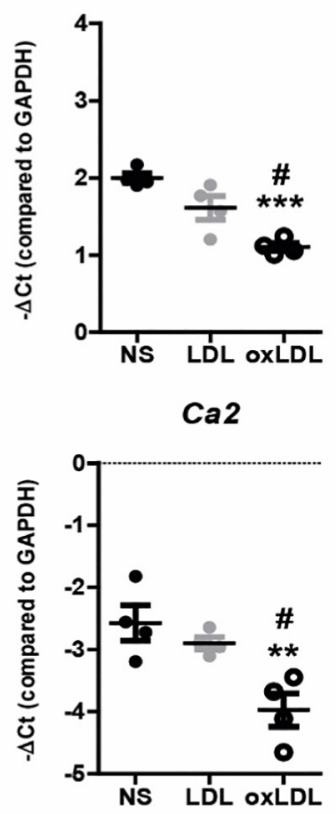

Calcr
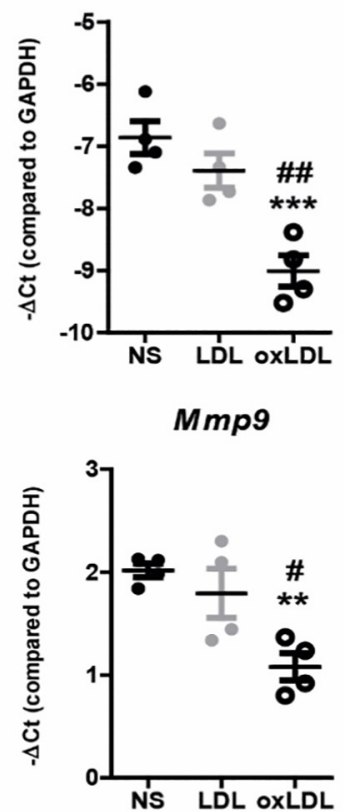

Ctsk

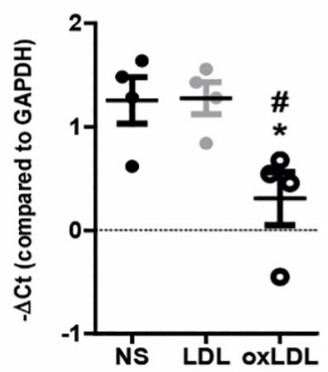

Adgre1

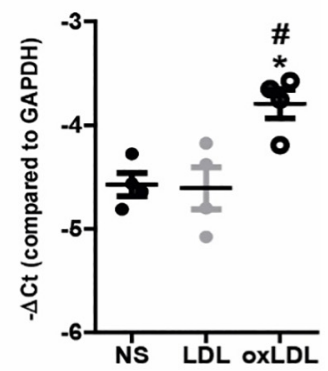

d)

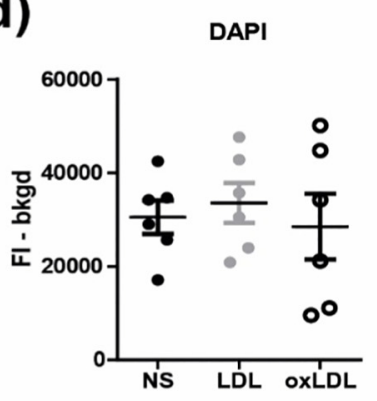


Fig. 4. oxLDL blocks the differentiation of mononuclear pre-osteoclasts into mature osteoclasts.

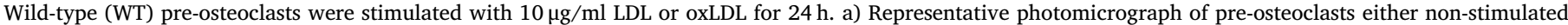

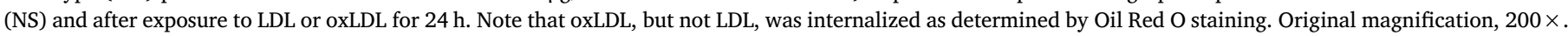

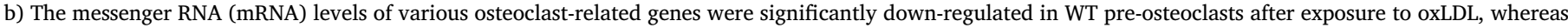

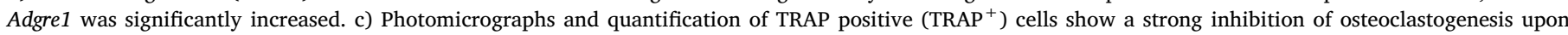

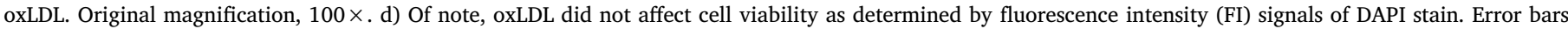

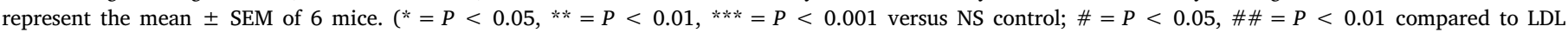
stimulated samples).

bone mass as determined by measuring the surface of the cortical bone in the arthritic knee joints (data not shown). Together, this suggests that Apoe deficiency and high LDL levels can interfere with osteoblastogenesis and bone formation, whereas bone resorption remains unaffected. In contrast, LDL receptor deficient $\left(L d l r^{-/-}\right)$mice, which represent another model of hypercholesterolemia, showed increased bone mass caused by impaired osteoclast-mediated bone resorption [45], which may be due to the fact that the LDL receptor is required to allow cholesterol uptake into cells under physiologic conditions [46].

Some studies have described that hypercholesterolemia and increased lipid oxidation reduce bone mass [47-49]. Discrepancies between our and previous findings may be explained by the fact that we studied $A p o e^{-/-}$mice that received a standard chow diet, while these previous studies used a high fat diet (HFD). HFD massively elevates LDL levels and its oxidation [47,49], thus contributing to increase of systemic inflammation that can affect bone formation [50,51].

Interestingly, although LDL levels as found in Apoe ${ }^{-/-}$mice fed a standard chow diet do not change bone destruction in a normal joint, in combination with synovial inflammation a strong reduction in development of bone destruction was observed that coincided with a significant reduction of osteoclasts on the cortical bone. This reduction was not due to a difference in OCP numbers since the numbers of $\mathrm{CD}_{11} \mathrm{~b}^{+} \mathrm{Ly}_{6 \mathrm{C}} \mathrm{high}^{\text {hig }}$ and $\mathrm{CD} 11 \mathrm{~b}^{-} \mathrm{Ly}_{6 \mathrm{C}}{ }^{\text {high }}$, the main OCPs, were not decreased in BM of arthritic Apoe $e^{-/-}$mice and their WT controls. This suggests that even though Apoe $e^{-/-}$mice developed decreased synovial inflammation [52] the generation of OCPs in the BM and their potential differentiation into osteoclasts in the knee joints is not affected. Interestingly, bone areas within the same knee joint that did not have direct contact with inflammatory cells (like the BM cortical bone) showed a similar amount of $\mathrm{TRAP}^{+}$osteoclasts between the two strains again indicating that high LDL levels alone without oxidation do not impair the number of osteoclasts. Therefore, we assume that enhanced LDL oxidation in the arthritic synovium of $\mathrm{Apoe}^{-/-}$mice may lead to a locally induced reduction of osteoclast numbers and diminished bone destruction.

As we could not detect oxLDL directly within the inflamed knee joints as the heterogeneity of oxLDL, due to different degrees as well as specific epitopes of oxidation, makes its detection in vivo very difficult $[37,53]$, we further investigated the effect of LDL/oxLDL in an in vitro osteoclastogenesis assay. Our findings strongly point out that oxLDL prevented the differentiation into multinucleated osteoclasts both when given in an early and later stage of osteoclastogenesis, thereby strongly reducing gene expression of osteoclast markers and resorption on biomimetic hydroxyapatite-like-coated plates. The decrease in osteoclastogenesis was not due to cell death since DAPI staining showed comparable numbers of cells in treated versus non-treated groups. In contrast to oxLDL, we observed no significant in vitro effect of LDL on osteoclast formation and activity and LDL only mildly reduced resorption. The latter may also be an oxLDL effect since the extended time frame of the cultures needed to investigate the resorption may have led to a partial oxidation of native LDL into oxLDL in the culture supernatant. Our findings are in line with the study of Mazière et al. [54] who also found that oxLDL blocks the in vitro differentiation of OCPs into osteoclasts. However, in contrast to their studies in which oxLDL was added to very early stages of OCPs we focused more on further differentiated pre-osteoclasts. The reason for this is that OCPs coming from the BM that enter the arthritic joint probably exhibit a more developed differentiation stage that is further steered by the inflammatory process within the inflamed synovium. Interestingly, Mazière et al. found that oxLDL blocks the in vitro differentiation of BM OCPs into osteoclasts upstream by inhibiting the RANK/RANKL signaling axis, which is crucial for induction of osteoclastogenesis [54]. In contrast, we found that stimulation with oxLDL when added later on in the differentiation process of osteoclastogenesis had no effects on the expression of RANK, but rather on some of the factors involved in the likewise important ITAM-mediated signaling pathway. The co-stimulatory pathway for osteoclastogenesis is triggered via phosphorylation of the ITAM domain present on the adapter molecules $\gamma$-chain which is paired with the receptors OSCAR, PIRA and Fc $\gamma$ Rs and DAP12 with TREM2 [55-57]. Of note, Oscar mRNA levels were sharply down-regulated in pre-osteoclasts exposed to oxLDL, implying that fewer OSCAR receptors are available to trigger the ITAM-pathway through the $\gamma$-chain. In contrast, Trem 2 mRNA was significantly up-regulated by oxLDL. However, as we find that its signaling molecule Tyrobp is strongly decreased, it is unlikely that the up-regulation of Trem2 leads to a greater activation of the co-stimulatory pathway of osteoclastogenesis.

Previous studies reported that TREM-2 expression steers the differentiation of M2-like macrophages [58,59]. Moreover, pre-osteoclasts exposed to oxLDL also showed increased mRNA levels of F4/80 which is a major macrophage marker $[60,61]$. These results indicate that cells exposed to oxLDL differentiate toward the macrophage rather than the osteoclast lineage despite high levels of RANKL and M-CSF. Moreover, Fc $\gamma$ RIII, which has been shown to inhibit osteoclastogenesis by sequestering the $\gamma$-chain and withholding its association with osteoclastinducer immune receptors like OSCAR [24], was significantly upregulated. It has to be mentioned that in this study we predominantly focused on the ITAM co-stimulatory signaling pathway for osteoclastogenesis and therefore cannot rule out that oxLDL interferes with other signaling pathways (i.e.: IL-6, TNF- $\alpha$ ) that are relevant for bone destruction in RA. Together, these observations substantiate the hypothesis that at later stages of osteoclastogenesis, oxLDL inhibits the differentiation into mature cells by interfering with the ITAM costimulatory pathway.

In another study, Dawodu et al. showed that oxLDL stimulation of human OCPs from peripheral blood impaired osteoclast activity via inhibition of CtsK secretion [62]. In our study, we also found that Ctsk but also Mmp9 mRNA levels were sharply decreased and may contribute to lowering of bone erosion. This makes it highly likely that oxLDL protects from bone erosion by inhibition of resorption of both inorganic and organic matrix.

Conflicting results have been reported in RA patients following cholesterol-lowering therapies. Whereas the use of statins is beneficial in managing the cardiovascular (CV) risk in RA patients [63-65], several studies have described the risk of developing RA during statin treatment in subjects prone to develop RA $[66,67]$. In line with this, experimental studies found that mice treated with statins showed accelerated onset of disease in a collagen-type II-induced arthritis (CIA) model [68]. Our study supports the hypothesis that lowering LDL levels may boost both initiation and progression of bone destruction locally within an inflamed joint.

In summary, our findings suggest that high levels of LDL in combination with inflammation in the knee joint leads to oxLDL that 
a)

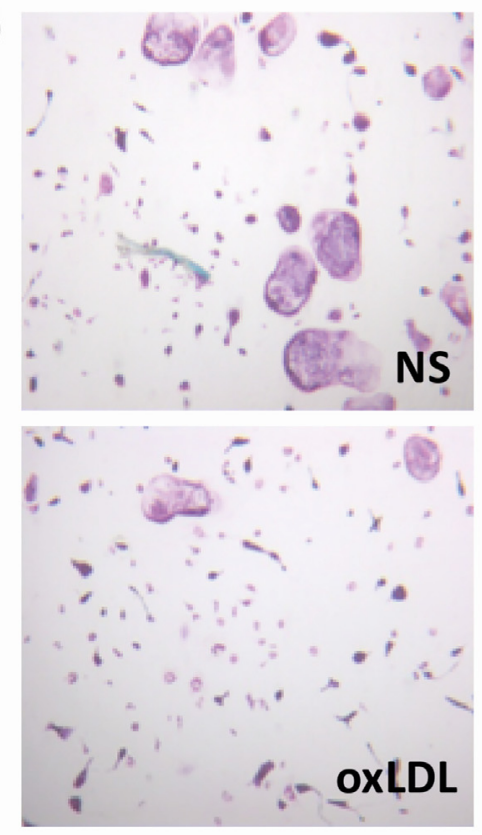

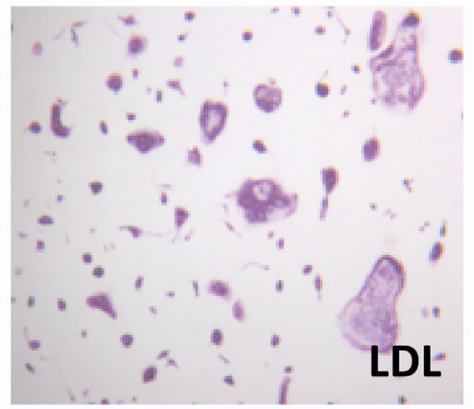

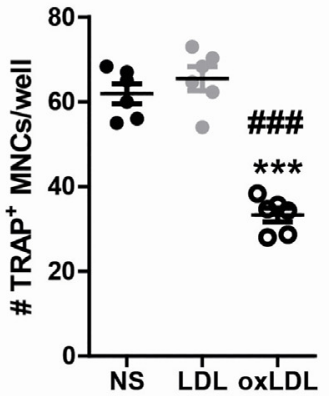

b)

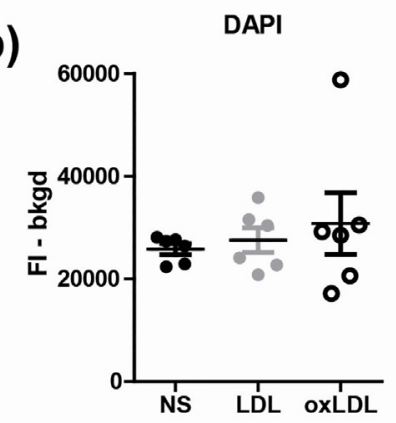

LDL

c) NS DAY 3

DAY 5

oxLDL
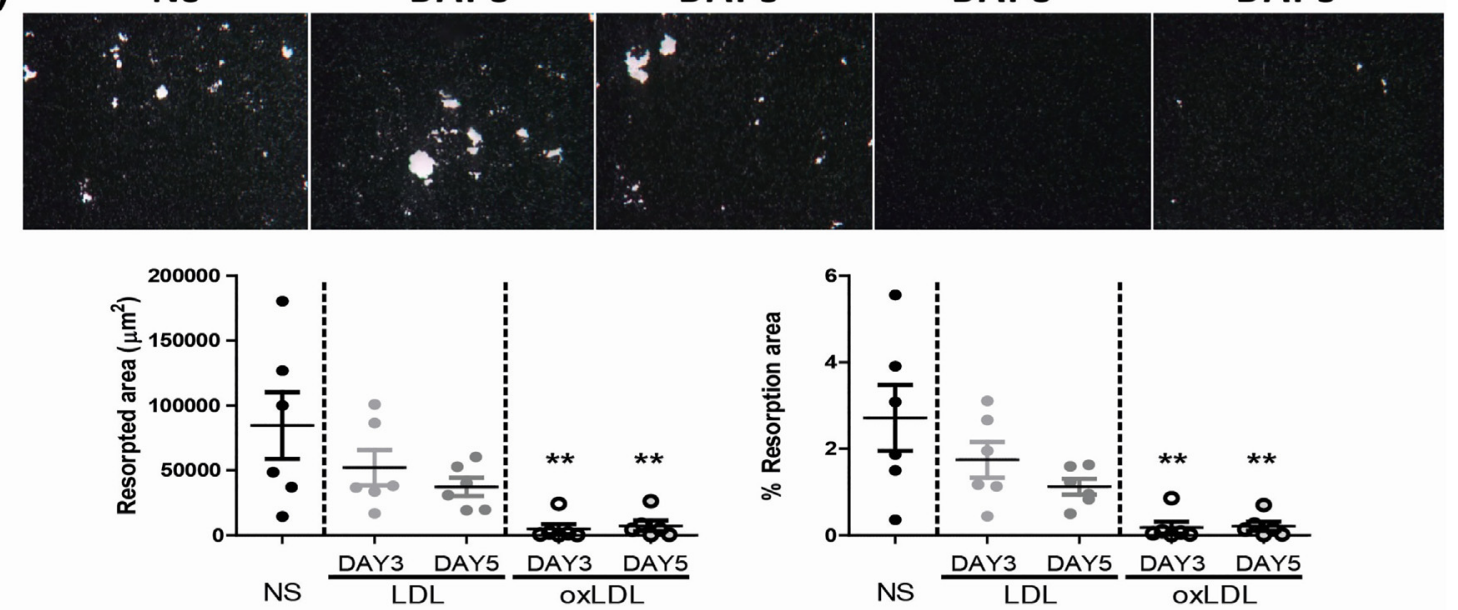

d)

TRAP activity

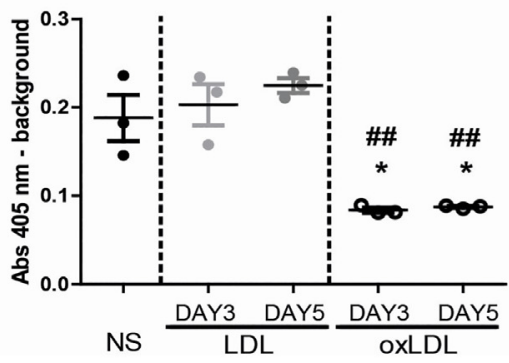

Fig. 5. oxLDL strongly restrains the formation of multinucleated osteoclasts and their resorptive activity at a later stage of differentiation. Wild-type (WT) multinucleated osteoclasts were stimulated with $10 \mu \mathrm{g} / \mathrm{ml} \mathrm{LDL}$ or oxLDL for $24 \mathrm{~h}$. a) Representative photomicrographs and quantification of TRAP positive (TRAP ${ }^{+}$) cells show a strong inhibition of osteoclastogenesis upon oxLDL, but not LDL stimulation. Original magnification, $100 \times$ b) Note that oxLDL did not affect cell viability as determined by fluorescence intensity (FI) signals of DAPI stain $(n=6$ mice). c) Representative photomicrographs and quantification of resorption on biomimetic hydroxyapatite-like-coated plates show a significant reduction of pit areas upon exposure of both pre-osteoclasts (day 3) and multinucleated osteoclasts (day 5) to oxLDL; of note, exposure to LDL reduced pit formation, though this reduction was not significant ( $\mathrm{n}=6$ mice). Original magnification, $100 \times$ d) Finally, TRAP activity in the culture supernatants of WT mature osteoclasts was strongly down-regulated both upon exposure of pre-osteoclasts (day 3) and multinucleated osteoclasts (day 5) to oxLDL, but not LDL ( $=3$ mice). Error bars represent the mean \pm SEM. $\left(*=P<0.05\right.$, ${ }^{* *}=P<0.01$, $* * * P<0.001$ compared to NS controls; \#\# $=P<0.01$, \#\#\# $=P<0.001$ compared to LDL stimulated samples). 
a)

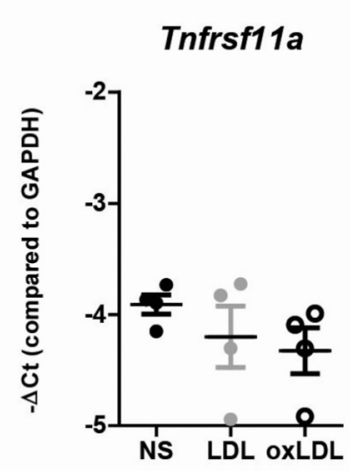

Csf1r

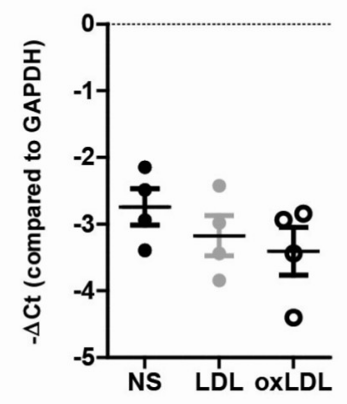

c)

Fcgr1

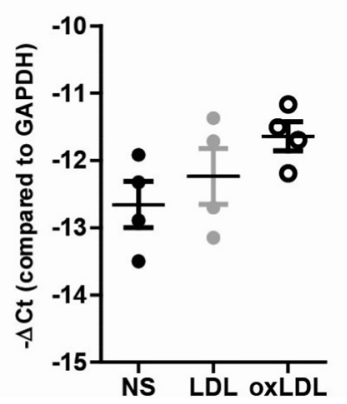

b)

Oscar

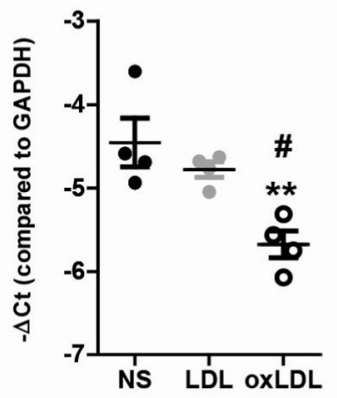

Trem2

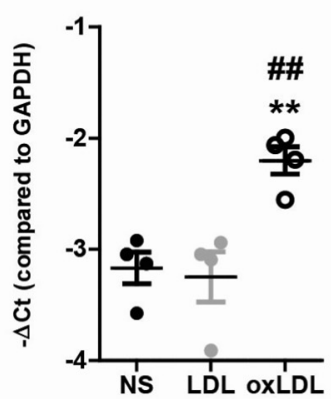

Fcgr2b

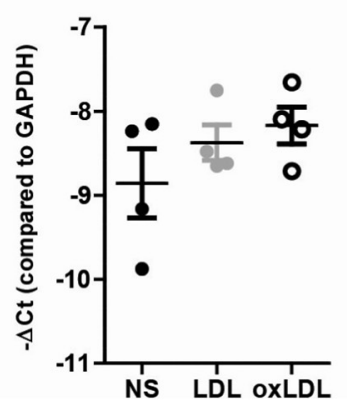

II2rg

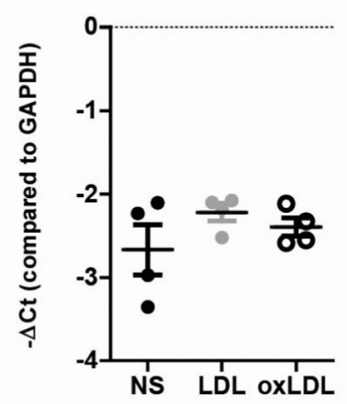

Tyrobp

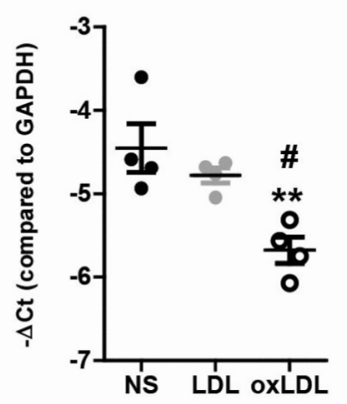

Pira

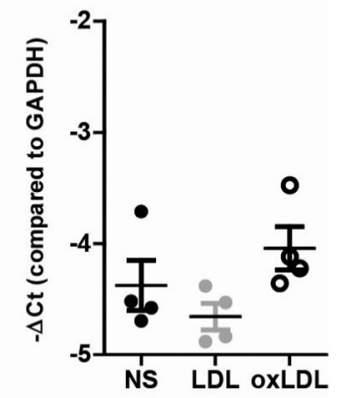

Pirb

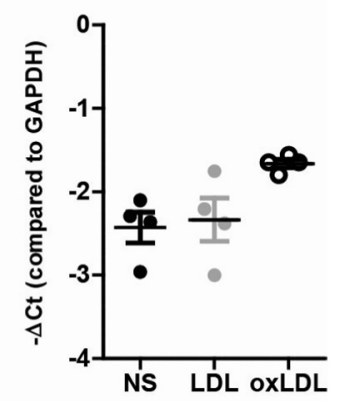

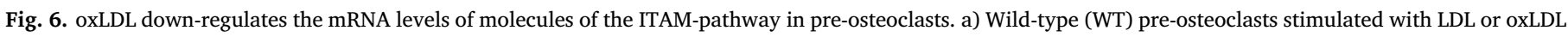

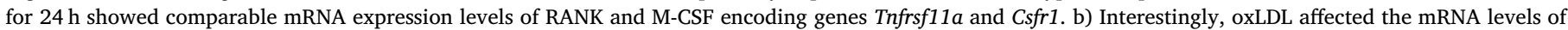

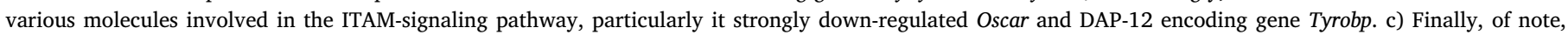

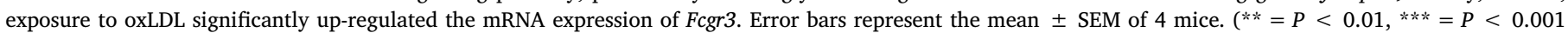
compared to NS controls; \# $=P<0.05$, \#\# $=P<0.01$, \#\#\# $=P<0.001$ compared to LDL stimulated samples).

inhibits bone destruction probably by suppressing osteoclast differentiation via decreased activation of the ITAM co-stimulatory pathway. Our study suggests that LDL-lowering therapies that are often used to reduce $\mathrm{CV}$ risk in RA patients should be used with caution since lowering of local levels of LDL within the inflamed joints may diminish the suppressing mechanism induced by oxLDL leading to increased osteoclastogenesis and bone destruction.

Supplementary data to this article can be found online at https:// doi.org/10.1016/j.bone.2019.115140.

\section{Acknowledgements}

The authors are thankful to Ineke Jansen (VU ACTA-Amsterdam) that kindly provided us with the biomimetic hydroxyapatite-like-coated plates for the assessment of bone mineral matrix dissolution.

\section{Funding}

This study was funded by Euroclast, a Marie Curie Initial Training network (FP7-People-2013-ITN: $\mathrm{N}^{0}$ 607447) and ReumaNederland NR: 16-1-402.

Declaration of competing interest

The authors disclose no competing interests. 


\section{References}

[1] G. Schett, E. Gravallese, Bone erosion in rheumatoid arthritis: mechanisms, diagnosis and treatment, Nat. Rev. Rheumatol. 8 (2012) 656-664.

[2] S.R. Goldring, Bone and joint destruction in rheumatoid arthritis: what is really happening? J Rheumatol Suppl 65 (2002) 44-48.

[3] E.M. Gravallese, Bone destruction in arthritis, Ann. Rheum. Dis. 61 (Suppl. 2) (2002) ii84-86.

[4] S.M. Jung, K.W. Kim, C.W. Yang, S.H. Park, J.H. Ju, Cytokine-mediated bone destruction in rheumatoid arthritis, J Immunol Res 2014 (2014) 263625.

[5] T. Braun, J. Zwerina, Positive regulators of osteoclastogenesis and bone resorption in rheumatoid arthritis, Arthritis Res Ther 13 (2011) 235.

[6] M. Asagiri, H. Takayanagi, The molecular understanding of osteoclast differentiation, Bone 40 (2007) 251-264.

[7] W.J. Boyle, W.S. Simonet, D.L. Lacey, Osteoclast differentiation and activation, Nature 423 (2003) 337-342.

[8] M. Seeling, et al., Inflammatory monocytes and Fcgamma receptor IV on osteoclasts are critical for bone destruction during inflammatory arthritis in mice, Proc. Natl. Acad. Sci. U. S. A. 110 (2013) 10729-10734.

[9] J.F. Charles, et al., Inflammatory arthritis increases mouse osteoclast precursors with myeloid suppressor function, J. Clin. Invest. 122 (2012) 4592-4605.

[10] T.N. Crotti, A.A. Dharmapatni, E. Alias, D.R. Haynes, Osteoimmunology: major and costimulatory pathway expression associated with chronic inflammatory induced bone loss, J Immunol Res 2015 (2015) 281287.

[11] L.B. Ivashkiv, Cross-regulation of signaling by ITAM-associated receptors, Nat. Immunol. 10 (2009) 340-347.

[12] M. Shinohara, et al., Tyrosine kinases Btk and Tec regulate osteoclast differentiation by linking RANK and ITAM signals, Cell 132 (2008) 794-806.

[13] T. Koga, et al., Costimulatory signals mediated by the ITAM motif cooperate with RANKL for bone homeostasis, Nature 428 (2004) 758-763.

[14] A. Mocsai, et al., The immunomodulatory adapter proteins DAP12 and Fc receptor gamma-chain (FcRgamma) regulate development of functional osteoclasts through the Syk tyrosine kinase, Proc. Natl. Acad. Sci. U. S. A. 101 (2004) 6158-6163.

[15] Y. Mori, et al., Inhibitory immunoglobulin-like receptors LILRB and PIR-B negatively regulate osteoclast development, J. Immunol. 181 (2008) 4742-4751.

[16] B. Nowak, M. Madej, A. Luczak, R. Malecki, P. Wiland, Disease activity, oxidized LDL fraction and anti-oxidized LDL antibodies influence cardiovascular risk in rheumatoid arthritis, Adv. Clin. Exp. Med. 25 (2016) 43-50.

[17] J. Wang, B. Hu, L. Kong, H. Cai, C. Zhang, Native, oxidized lipoprotein(a) and lipoprotein(a) immune complex in patients with active and inactive rheumatoid arthritis: plasma concentrations and relationship to inflammation, Clin. Chim. Acta 390 (2008) 67-71.

[18] C.P. Chung, et al., Prevalence of the metabolic syndrome is increased in rheumatoid arthritis and is associated with coronary atherosclerosis, Atherosclerosis 196 (2008) $756-763$.

[19] S.H. Zhang, R.L. Reddick, J.A. Piedrahita, N. Maeda, Spontaneous hypercholesterolemia and arterial lesions in mice lacking apolipoprotein E, Science 258 (1992) $468-471$.

[20] M. Tani, et al., The influence of apoE-deficiency and LDL-receptor-deficiency on the HDL subpopulation profile in mice and in humans, Atherosclerosis 233 (2014) 39-44.

[21] W. de Munter, et al., Cholesterol accumulation caused by low density lipoprotein receptor deficiency or a cholesterol-rich diet results in ectopic bone formation during experimental osteoarthritis, Arthritis Res Ther 15 (2013) R178.

[22] J.P. Rhoads, et al., Oxidized low-density lipoprotein immune complex priming of the Nlrp3 inflammasome involves TLR and FcgammaR cooperation and is dependent on CARD9, J. Immunol. 198 (2017) 2105-2114.

[23] B. ten Harkel, et al., The foreign body giant cell cannot resorb bone, but dissolves hydroxyapatite like osteoclasts, PLoS One 10 (2015) e0139564.

[24] T. Negishi-Koga, et al., Immune complexes regulate bone metabolism through FcRgamma signalling, Nat. Commun. 6 (2015) 6637.

[25] C.N. Manjunath, J.R. Rawal, P.M. Irani, K. Madhu, Atherogenic dyslipidemia, Indian J Endocrinol Metab 17 (2013) 969-976.

[26] A. Bag-Ozbek, J.T. Giles, Inflammation, adiposity, and atherogenic dyslipidemia in rheumatoid arthritis: is there a paradoxical relationship? Curr Allergy Asthma Rep 15 (2015) 497.

[27] Y.J. Park, C.S. Cho, P. Emery, W.U. Kim, LDL cholesterolemia as a novel risk factor for radiographic progression of rheumatoid arthritis: a single-center prospective study, PLoS One 8 (2013) e68975.

[28] A.M. Archer, et al., ApoE deficiency exacerbates the development and sustainment of a semi-chronic K/BxN serum transfer-induced arthritis model, J. Transl. Med. 14 (2016) 170.

[29] W. de Munter, et al., High LDL levels lead to increased synovial inflammation and accelerated ectopic bone formation during experimental osteoarthritis, Osteoarthr. Cartil. 24 (2016) 844-855.

[30] W. de Munter, et al., Synovial macrophages promote TGF-beta signaling and protect against influx of S100A8/S100A9-producing cells after intra-articular injections of oxidized low-density lipoproteins, Osteoarthr. Cartil. 25 (2017) 118-127.

[31] J.M. Quinn, et al., Transforming growth factor beta affects osteoclast differentiation via direct and indirect actions, J. Bone Miner. Res. 16 (2001) 1787-1794.

[32] H. Takai, et al., Transforming growth factor-beta stimulates the production of osteoprotegerin/osteoclastogenesis inhibitory factor by bone marrow stromal cells, J. Biol. Chem. 273 (1998) 27091-27096.

[33] B.H. Hahn, J. Grossman, W. Chen, M. McMahon, The pathogenesis of atherosclerosis in autoimmune rheumatic diseases: roles of inflammation and dyslipidemia, J. Autoimmun. 28 (2007) 69-75.

[34] F. Oliviero, et al., A comparative study of serum and synovial fluid lipoprotein levels in patients with various arthritides, Clin. Chim. Acta 413 (2012) 303-307.

[35] Y.S. Bae, et al., Macrophages generate reactive oxygen species in response to minimally oxidized low-density lipoprotein: toll-like receptor 4- and spleen tyrosine kinase-dependent activation of NADPH oxidase 2, Circ. Res. 104 (2009) 210-218 221 p following 218.

[36] O.J. Lara-Guzman, et al., Oxidized LDL triggers changes in oxidative stress and inflammatory biomarkers in human macrophages, Redox Biol. 15 (2018) 1-11.

[37] I. Levitan, S. Volkov, P.V. Subbaiah, Oxidized LDL: diversity, patterns of recognition, and pathophysiology, Antioxid. Redox Signal. 13 (2010) 39-75.

[38] E.J. Schaefer, et al., Familial apolipoprotein E deficiency, J. Clin. Invest. 78 (1986) 1206-1219.

[39] A.A. Pendse, J.M. Arbones-Mainar, L.A. Johnson, M.K. Altenburg, N. Maeda, Apolipoprotein E knock-out and knock-in mice: atherosclerosis, metabolic syndrome, and beyond, J. Lipid Res. 50 (2009) S178-S182 Suppl.

[40] H. Zhang, L.M. Wu, J. Wu, Cross-talk between apolipoprotein E and cytokines, Mediat. Inflamm. 2011 (2011) 949072.

[41] K. Ali, M. Middleton, E. Pure, D.J. Rader, Apolipoprotein E suppresses the type inflammatory response in vivo, Circ. Res. 97 (2005) 922-927.

[42] K. Li, D. Ching, F.S. Luk, R.L. Raffai, Apolipoprotein E enhances microRNA-146a in monocytes and macrophages to suppress nuclear factor-kappaB-driven inflammation and atherosclerosis, Circ. Res. 117 (2015) e1-e11.

[43] A.F. Schilling, et al., Increased bone formation in mice lacking apolipoprotein E, J. Bone Miner. Res. 20 (2005) 274-282.

[44] I.G. Nikolov, et al., Chronic kidney disease bone and mineral disorder (CKD-MBD) in apolipoprotein E-deficient mice with chronic renal failure, Bone 47 (2010) $156-163$.

[45] M. Okayasu, et al., Low-density lipoprotein receptor deficiency causes impaired osteoclastogenesis and increased bone mass in mice because of defect in osteoclastic cell-cell fusion, J. Biol. Chem. 287 (2012) 19229-19241.

[46] P.L. Yeagle, Lipid regulation of cell membrane structure and function, FASEB J. 3 (1989) 1833-1842.

[47] H. Hirasawa, et al., ApoE gene deficiency enhances the reduction of bone formation induced by a high-fat diet through the stimulation of p53-mediated apoptosis in osteoblastic cells, J. Bone Miner. Res. 22 (2007) 1020-1030.

[48] F. Pirih, et al., Adverse effects of hyperlipidemia on bone regeneration and strength, J. Bone Miner. Res. 27 (2012) 309-318.

[49] A.P. Sage, et al., Hyperlipidemia induces resistance to PTH bone anabolism in mice via oxidized lipids, J. Bone Miner. Res. 26 (2011) 1197-1206.

[50] M. Almeida, E. Ambrogini, L. Han, S.C. Manolagas, R.L. Jilka, Increased lipid oxidation causes oxidative stress, increased peroxisome proliferator-activated receptorgamma expression, and diminished pro-osteogenic Wnt signaling in the skeleton, $\mathrm{J}$. Biol. Chem. 284 (2009) 27438-27448.

[51] M.S. Huang, et al., Hyperlipidemia impairs osteoanabolic effects of PTH, J. Bone Miner. Res. 23 (2008) 1672-1679.

[52] G. Ascone, et al., High LDL-C levels attenuate onset of inflammation and cartilage destruction in antigen-induced arthritis, Clin. Exp. Rheumatol. (2019).

[53] T. Wu, et al., Is plasma oxidized low-density lipoprotein, measured with the widely used antibody 4E6, an independent predictor of coronary heart disease among U.S. men and women? J. Am. Coll. Cardiol. 48 (2006) 973-979.

[54] C. Maziere, et al., Oxidized low density lipoprotein decreases Rankl-induced differentiation of osteoclasts by inhibition of Rankl signaling, J. Cell. Physiol. 221 (2009) 572-578

[55] A.D. Barrow, et al., OSCAR is a collagen receptor that costimulates osteoclastogenesis in DAP12-deficient humans and mice, J. Clin. Invest. 121 (2011) 3505-3516.

[56] J.H. Kim, et al., Upstream stimulatory factors regulate OSCAR gene expression in RANKL-mediated osteoclast differentiation, J. Mol. Biol. 383 (2008) 502-511.

[57] M.B. Humphrey, M.C. Nakamura, A comprehensive review of immunoreceptor regulation of osteoclasts, Clin. Rev. Allergy Immunol. 51 (2016) 48-58.

[58] I.R. Turnbull, et al., Cutting edge: TREM-2 attenuates macrophage activation, J. Immunol. 177 (2006) 3520-3524.

[59] K. Wu, et al., TREM-2 promotes macrophage survival and lung disease after respiratory viral infection, J. Exp. Med. 212 (2015) 681-697.

[60] J.M. Austyn, S. Gordon, F4/80, a monoclonal antibody directed specifically against the mouse macrophage, Eur. J. Immunol. 11 (1981) 805-815.

[61] L. Morris, C.F. Graham, S. Gordon, Macrophages in haemopoietic and other tissues of the developing mouse detected by the monoclonal antibody F4/80, Development 112 (1991) 517-526.

[62] D. Dawodu, et al., oxLDL inhibits differentiation and functional activity of osteoclasts via scavenger receptor-a mediated autophagy and cathepsin K secretion, Sci. Rep. 8 (2018) 11604.

[63] T. Zegkos, G. Kitas, T. Dimitroulas, Cardiovascular risk in rheumatoid arthritis: assessment, management and next steps, Ther Adv Musculoskelet Dis 8 (2016) 86-101.

[64] A.M. Chodara, A. Wattiaux, C.M. Bartels, Managing cardiovascular disease risk in rheumatoid arthritis: clinical updates and three strategic approaches, Curr. Rheumatol. Rep. 19 (16) (2017).

[65] S. Soulaidopoulos, E. Nikiphorou, T. Dimitroulas, G.D. Kitas, The role of statins in disease modification and cardiovascular risk in rheumatoid arthritis, Front Med (Lausanne) 5 (24) (2018).

[66] H.J. de Jong, et al., Use of statins is associated with an increased risk of rheumatoid arthritis, Ann. Rheum. Dis. 71 (2012) 648-654.

[67] S.S. Jick, H. Choi, L. Li, I.B. McInnes, N. Sattar, Hyperlipidaemia, statin use and the risk of developing rheumatoid arthritis, Ann. Rheum. Dis. 68 (2009) 546-551.

[68] R.J. Vandebriel, et al., Statins accelerate the onset of collagen type II-induced arthritis in mice, Arthritis Res Ther 14 (2012) R90. 